\title{
Cautionary Note About the Herfindahl-Hirschman Index of Market (Industry) Concentration \\ Tarald O. Kvålseth
}

\begin{abstract}
The Herfindahl-Hirschman index $(H H I)$ appears to be the most widely used index of market or industrial concentration. It is a summary measure that indicates the degree of competition, market power, and efficiency within a market or an industry. The HHI is also used by government agencies when evaluating potential violation of antitrust laws and regulations. As emphasized in this paper, and in spite of its several desirable properties, $\mathrm{HH}$ l has one serious limitation: it lacks the value-validity property. Lacking this property, caution has to be exercised when using $\mathrm{HHI}$ in order to avoid invalid and misleading results and conclusions. A corrected index is developed as a simple reformulation of $\mathrm{HHI}$. Since this new index formulation meets the conditions imposed by the valuevalidity property to a high degree of approximation, and has other desirable properties comparable to those of $\mathrm{HHI}$, the corrected index can safely be used to make various types of comparisons that are true and valid representations of market (industry) concentration. Numerical data are provided to support and exemplify the use of the corrected index.
\end{abstract}

KEY WORDS: $\quad$ Market concentration, Industrial concentration, Herfindahl-Hirschman index, Value validity.

JEL Classification: L16, L19, L40, C18.

University of Minnesota, Minneapolis, MN 55455, USA

Departments of Mechanical Engineering and Industrial \& Systems Engineering

\section{Introduction}

Market concentration, which is also often referred to as industrial concentration, refers to the extent to which the market shares are concentrated among the largest firms within a market (industry). Such concentration of economic activity in terms of output, sales, assets, employment or other variables may serve as an indicator of the extent of the competition, efficiency, and market power. Trends in market concentration are being monitored by the business community and by agencies such as the U.S. Department of Justice (DOJ) and the Federal Trade Commission (FTC) in the case of potential antitrust concern.

A number of different measures or indices of market (industry) concentration have been proposed over the years (see, e.g., Hannah \& Kay 1977, Ch. 4; Bikker \& Haaf 2002; Ginevičius \& Čirba 2007; and Ukav 2017

Correspondence concerning this article should be addressed to: Tarald O. Kvålseth, Departments of Mechanical Engineering and Industrial \& Systems Engineering, University of Minnesota, Minneapolis, MN 55455, USA. E-mail: kvals001@umn.edu for such a review). Other alternatives have been considered more recently by, for example, Boone (2008) and Anbarci and Katzman (2015). However, the single most popular concentration measure is the Herfindahl-Hirschman index (HHI) after Herfindahl (1950) and Hirschman (1945). In fact, HHI has become one of the most popular summary statistics in general, especially since the DOJ and FTC began using the $\mathrm{HHI}$ instead of the concentration ratio as part of the Horizontal Merger Guidelines in 1982 and in subsequent editions $(1984,1992,1997,2010)$. Although the HHI and the concentration ratio are somewhat related in terms of boundary values, $H H I$ is generally preferred because of its superior properties (Kvålseth 2018). The $\mathrm{HHI}$ is also used by the European Commission (EC) as a means of identifying potential competitive concerns raised by mergers (Gaughan 2011, pp. 117-122; European Union 2004). Calkins (1983) provides an interesting account of $\mathrm{HHI}$ from a legal perspective.

Nevertheless, in spite of its popularity and several desirable properties, the $H H I$ lacks one particular property that is necessary for making certain interpre- 
tations and comparisons of concentration values. It is the purpose of the present paper to discuss this important issue and caution against invalid uses of $\mathrm{HHI}$. Otherwise, as pointed out, the use of HHI may lead to incorrect and misleading results, interpretations, or conclusions.

In order to avoid such potential problems and misuses of $H H I$, this paper develops a corrected index as a simple function of $\mathrm{HHI}$ subject to a constraint. The new formulation is supported by numerical data.

\section{Index Properties}

\subsection{Definitions and Notation}

Let $s_{1}, \ldots, s_{n}$ denote the respective market shares (as proportions or percentages) of the $n$ firms within a market (industry) so that $\sum_{i=1}^{n} s_{i}=1$ or $100 \%$. The HerfindahlHirschman index, most commonly denoted by HHI, is then defined as

$$
H H I=\sum_{i=1}^{n} s_{i}^{2}
$$

The potential values of $H H I$ range from $1 / n$ to 1 (or $10,000 / n$ to 10,000 if the $S_{i}$ are considered in terms of percentages). Since very small market shares are assigned little weight in the computation of $\mathrm{HHI}$ and are often not reported in the market-share data, those $\boldsymbol{S}_{\boldsymbol{i}}$ are often not included in (1).

Furthermore, let $C$ denote a generic concentration measure or index. The value of $C$ for the market-share distribution $S_{n}=\left(s_{1}, \ldots, S_{n}\right)$ is most appropriately denoted by $C\left(S_{n}\right)$.

However, for notational simplicity and when there is no chance of ambiguity, $C$ will be used here as both a measure and its numerical value.

\subsection{General requirements}

Properties of a concentration measure $C$, whether necessary or desirable, have been extensively discussed over the years (e.g., Hall \& Tideman 1967; Hannah \& Kay 1977, Ch. 4; Encaoua \& Jacquemin 1980; Chakravarty \&Eichhorn 1991). Such necessary properties of $C$ may be summarized as follows: (P1) Continuity: $C$ is a continuous function of all the $s_{i}(i=1, \ldots, n)$.

(P2) Symmetry: $C$ is (permutation) symmetric in $s_{1}, \ldots, s_{n}$.

(P3) Zero-indifference: $C$ is unaffected if one or more firms with zero market share enter or leave the market, i.e., $C\left(s_{1}, \ldots, s_{n}, 0, \ldots, 0\right)=C\left(s_{1}, \ldots, s_{n}\right)$.
(P4) Schur-convexity: $C$ is strictly Schur-convex.

(P5) Value validity: $\mathrm{C}$ has value validity (as discussed below).

The continuity Property (P1) ensures that small changes in some of the $s_{i}(i=1, \ldots, n)$ result in only a small change in the value of $C$. Property (P2) simply states that $C$ is invariant with respect to the order in which the original $s_{i}(i=1, \ldots, n)$ are given. According to Property (P3), the addition or deletion of one or more $s_{i}=0$ components to or from the distribution $S_{n}=\left(s_{1}, \ldots, S_{n}\right)$ has no effect on the value of $C$. Property (P3) together with (P1) has the effect of slightly decreasing the value of $C$ if one or more additional small firms enter the market (or the reverse effect if the small firms leave the market). This particular (P3) also shows the fundamental difference between a measure of concentration and one of inequality (Chakravarty and Eichhorn 1991).

The strict Schur-convexity property (P4) implies that, if the components of the market-share distribution $S_{n}=\left(s_{1}, \ldots, s_{n}\right)$ are "more nearly equal" or "less spread out" than are the components of another market-share distribution $R_{n}=\left(r_{1}, \ldots, r_{n}\right)$, then $C\left(S_{n}\right)<C\left(R_{n}\right)$ In terms of majorization theory, the more precise meaning is that $S_{n}$ is majorized by $R_{n}$ so that, since $C$ is strictly Schur-convex, $C\left(S_{n}\right)<C\left(R_{n}\right)$ (Marshall et al., 2011,). This implication or condition, which economists refer to as the Dalton condition or the PigouDalton condition, also means that $C$ has the transfer property and preserves the Lorenz order (Marshall et al., 2011, pp. 5-8, 560, 712-723).

Various implications arise from these specific properties. For example, Properties (P3) and (P4) imply that a merger between a smaller firm and a larger one will cause the value of $C$ to increase. Similarly, in the case of markets or industries with firms of equal size (i.e., $s_{i}=1 / n$ for $i=1, \ldots, n$ ), Properties (P3)(P4) imply that the value of $C$ decreases with increasing $n$.

There would appear to be no particular controversy about Properties (P1)-(P4). They all seem to be intuitively reasonable and do not impose undue restriction on $C$. However, another property suggested by some, the so-called replication property, needs to be mentioned. If $C$ has this property, then $C\left(S_{n} / k\right)=C\left(S_{n}\right) / k$. That is, if the market share $S_{i}$ of each firm within a market (industry) is split up into $k$ equal parts $s_{i} / k, \ldots, s_{i} / k$ for $i=1, \ldots, n$, then concentration should be reduced by a multiplicative factor of $1 / k$. This property would seem to be primarily one of convenience and perhaps somewhat intuitive. However, it can certainly be argued that this is an 
unnecessary and unduly restrictive property. It is also entirely unrealistic for any real, practical situation. Also, importantly, the replication property is inconsistent with the value-validity property discussed next.

\subsection{Value Validity}

The value-validity Property (P5) means that all potential values of $C$ provide numerical representations of the size (extent) of the concentration characteristic that are true, realistic, or valid with respect to some acceptable criterion. The concept of value-validity has been quite extensively discussed by Kvålseth (2015) and is only briefly outlined here with respect to concentration.

It is generally considered that a concentration index should take on its extremal values for the following two distributions:

$S_{n}^{0}=\left(\frac{1}{n}, \ldots, \frac{1}{n}\right), S_{n}^{1}=(1,0, \ldots, 0)$.

These two distributions are members of the lambda distribution:

$S_{n}^{\lambda}=\left(\lambda+\frac{1-\lambda}{n}, \frac{1-\lambda}{n}, \ldots, \frac{1-\lambda}{n}\right), \quad 0 \leq \lambda \leq 1$

where $\lambda$ is some real-valued parameter ( $1-\lambda$ is used instead of $\lambda$ as a more convenient form when dealing with concentration) (Kvålseth 2011). The $\lambda$ is basically a concentration parameter with $\lambda=0$ and $\lambda=1$ as in (2) for minimum and maximum concentration, respectively. The $S_{n}^{\lambda}$ in (3) is seen to be the following linear function or weighted arithmetic mean of $S_{n}^{0}$ and $S_{n}^{1}$ in (2):

$S_{n}^{\lambda}=\lambda S_{n}^{1}+(1-\lambda) S_{n}^{0}, 0 \leq \lambda \leq 1$

Then, in order for the linearity in (4) to be transferred to the index $C$, it is necessary that the following linear formulation holds:

$$
C\left(S_{n}^{\lambda}\right)=\lambda C\left(S_{n}^{1}\right)+(1-\lambda) C\left(S_{n}^{0}\right)
$$

The requirement in (5) is also supported by a criterion involving distances between the distributions in (2) and (3). Thus, consider the normalized Euclidean distances:

$$
C^{*}\left(S_{n}^{\lambda}\right)=\frac{\left|C\left(S_{n}^{\lambda}\right)-C\left(S_{n}^{0}\right)\right|}{\left|C\left(S_{n}^{1}\right)-C\left(S_{n}^{0}\right)\right|}, d^{*}\left(S_{n}^{\lambda}\right)=\frac{d\left(S_{n}^{\lambda}, S_{n}^{0}\right)}{d\left(S_{n}^{1}, S_{n}^{0}\right)}=\lambda
$$

where $C^{*}\left(S_{n}^{\lambda}\right)$ and $d^{*}\left(S_{n}^{\lambda}\right)$ belong to the interval $[0,1]$. The proposition that

$C^{*}\left(S_{n}^{\lambda}\right)=d^{*}\left(S_{n}^{\lambda}\right)=\lambda$

results in (5). Furthermore, for any given market-share distribution $S_{n}=\left(s_{1}, \ldots, S_{n}\right)$, there is necessarily one unique $S_{n}^{\lambda}$ for which $C^{*}\left(S_{n}^{i}\right)=C^{*}\left(S_{n}\right)$, so that (7) should also apply to $S_{n}$, at least as a good approximation, as follows:

$C^{*}\left(S_{n}\right) \approx d^{*}\left(S_{n}\right)=\sqrt{\frac{n \sum_{i=1}^{n} s_{i}^{2}-1}{n-1}}=\frac{n}{\sqrt{n-1}} \sigma_{n}$

where $\sigma_{n}$ is the standard deviation of $s_{1}, \ldots, s_{n}$ (with devisor $n$ ). Consequently, (5) (or (7)) and (8) become the value-validity conditions for $C$.

As mentioned above, the replication property is inconsistent with the value-validity property. This can be simply proved as follows. Consider that $f(n) \leq C\left(S_{n}\right) \leq C\left(S_{n}^{1}\right)$ for any $S_{n}$ where $C\left(S_{n}^{1}\right)=1$ and $f$ is a strictly decreasing function of $n(f(n)=1 / n$ in the case of HHI in (1)). If $S_{n} / k$ denotes the market-share distribution $S_{n}=\left(s_{1}, \ldots, s_{n}\right)$ after each $s_{i}$ has been split into $k$ equal parts so that $S_{n}^{0} / k=(1 / k n, \ldots, 1 / k n)$ and $S_{n}^{1} / k=(1 / k, \ldots, 1 / k, 0, \ldots, 0)$, then the value-validity condition in (8) can be expressed as

$C\left(S_{n} / k\right) \approx(1-f(k n)) \sqrt{\frac{n \sum_{i=1}^{n} s_{i}^{2}-1}{n-1}}+f(k n)$.

However, the expression in (9) is different from the replication expression $C\left(S_{n} / k\right)=C\left(S_{n}\right) / k$ so that $C$ cannot have both the replication property and the value-validity property.

\section{Assessment of HHI}

It is clear from its expression in (1) that $H H I$ has Properties (P1)-(P3). It is also strictly Schur-convex (Property (P4)) (Marshall et al., 2011, pp. 138-139). Besides those properties, the $H H I$ has the desirable property, as an advantage over other indices, of emerging from a standard model of market performance (e.g., Martin, 2002, pp. 337-338). 
However, $\mathrm{HHI}$ does not have the value-validity Property (P5), which requires caution when using this index as will be explained. Specifically, for the distribution in (3),

$H H I\left(S_{n}^{\lambda}\right)=\lambda^{2} H H I\left(S_{n}^{1}\right)+\left(1-\lambda^{2}\right) H H I\left(S_{n}^{0}\right)=\left(1-\frac{1}{n}\right) \lambda^{2}+\frac{1}{n}$

where $H H I\left(S_{n}^{0}\right)$ and $H H I\left(S_{n}^{1}\right)$ are the values of $H H I$ for the particular distributions in (2) involving the minimal and maximal concentration, respectively, for any given $n$. With $\operatorname{HHI}\left(S_{n}^{0}\right)=1 / n$ and $H H I\left(S_{n}^{1}\right)=1$, the normalized form of $\operatorname{HHI}\left(S_{n}^{\lambda}\right)$ becomes

$\operatorname{HHI}^{*}\left(S_{n}^{\lambda}\right)=\frac{H H I\left(S_{n}^{\lambda}\right)-1 / n}{1-1 / n}=\lambda^{2}$.

It is clear from (10) or (11) that HHI fails to meet the value-validity conditions in (5) or (7). Furthermore from the expressions in (1) and (8), it is clear that HHI cannot meet the condition in (8).

With $C\left(S_{n}^{0}\right)=1 / n$ and $C\left(S_{n}^{1}\right)=1$, as in the case of $H H I$, (5) requires that

$C\left(S_{n}^{\lambda}\right)=\left(1-\frac{1}{n}\right) \lambda+\frac{1}{n}$.

Comparison between (10) and (12) shows that HHI understates the true extent of the concentration. Specifically,

$C\left(S_{n}^{\lambda}\right)-H H I\left(S_{n}^{\lambda}\right)=\left(1-\frac{1}{n}\right) \lambda(1-\lambda)$

which increases with $n$ for any given $\lambda$ and reaches a maximum when $\lambda=1 / 2$ for any given $n$. Similarly, in terms of the normalized indices in (7) and (11),

$C^{*}\left(S_{n}^{\lambda}\right)-H H I^{*}\left(S_{n}^{\lambda}\right)=\lambda(1-\lambda)$

which reaches its maximum for $\lambda=1 / 2$ as in the case of (13). Such value understatement by HHI can indeed be substantial when considered in absolute terms as in (13)-(14) or in relative terms.

As a simple numerical illustration, consider the case where $n=2$, and the lambda distribution in (3) for which $S_{2}^{\lambda}=[(1+\lambda) / 2,(1-\lambda) / 2]$, with $S_{2}^{0}=(1 / 2,1 / 2)$ and $S_{2}^{1}=(1,0)$ being the extreme cases in (2). For, say, $\lambda=0.5$ each component of $S_{2}^{0.5}=(0.75,0.25)$ is the same distance from the corresponding components of $S_{2}^{0}$ and $S_{2}^{1}$ so that the normalized value of a concentration measure should be $1 / 2$ according to (7) and as seems intuitively entirely reasonable. However, $\operatorname{HHI}^{*}(0.75,0.25)=0.25$, which is only half the value required by (7). Similarly, for example, $H_{H} I^{*}\left(S_{2}^{0.9}\right)=H H I^{*}(0.95,0.05)=0.81<\lambda=0.90$ and $H H I^{*}\left(S_{2}^{0.1}\right)=H H I^{*}(0.55,0.45)=0.01<\lambda=0.10$.

The same improper or unrealistic result $\operatorname{HHI}^{*}\left(S_{n}^{0.5}\right)=0.25$ is obtained for all $n$ as compared to 0.50 required by the value-validity condition in (7).

Such clear lack of value validity by the $H H I$ raises concern about the use of this index. However, because of its other desirable properties, the following caution would seem to be a prudent one: $H H I$ is appropriate for making order ("greater than" or "smaller than") comparisons of concentration values, but should not be used for comparisons of differences in concentration values. That is, if $h_{1}, h_{2}$, etc. denote values of $H H I$ for different market-share distributions (for different markets or for different times of the same market), a comparison such as $h_{1}>h_{2}$ is probably a safe comparison and true representation of the real market concentration characteristic. Other concentration measures with similar properties could be expected to provide similar order-comparison results. However, difference comparisons such as $h_{1}-h_{2}>h_{3}-h_{4}$ or $\left(h_{1}-h_{2}\right) / h_{2}>\left(h_{3}-h_{4}\right) / h_{4}$ cannot be expected to provide true or realistic representations of real market conditions since $H H I$ lacks the types of numerical values that are conditional on the value-validity property. In fact, such difference comparisons based on different concentration measures with similar properties, but lacking value-validity, can yield inconsistent and entirely opposite results.

As an illustration of such inconsistency or reversal of results, consider the square root of $\mathrm{HHI}$, with $\sqrt{H H I}$ having the same properties (P3)-(P4) as HHI. Of course, $\sqrt{H H I}=\sqrt{1 / n}$ versus $H H I=1 / n$ for the marketshare distribution $S_{n}^{0}$ in (2). Let three values of $H H I$ be ordered such that $h_{1}>h_{2}>h_{3}$.

It is a well-known property of power means that $\left(h_{1}+h_{3}\right) / 2>\left[\left(\sqrt{h_{1}}+\sqrt{h_{3}}\right) / 2\right]^{2}$ (e.g., Marshall et al., 2011, pp. 139-141). Thus, for any $h_{2}$ such that

$\frac{h_{1}+h_{3}}{2}>h_{2}>\left(\frac{\sqrt{h_{1}}+\sqrt{h_{3}}}{2}\right)^{2}$

it follows that

$h_{1}-h_{2}>h_{2}-h_{3}$

$\sqrt{h_{1}}-\sqrt{h_{2}}<\sqrt{h_{2}}-\sqrt{h_{3}}$ 
Table 1

Values of the Indices in (10), (12), and (19)-(20) for the Lambda Distribution in (3) with Varying $\lambda$ and $n$

\begin{tabular}{|c|c|c|c|c|c|c|}
\hline$\lambda$ & $n$ & $H H I$ & $C$ & $\sqrt{H H I}$ & $H H I_{0}$ & $H H I^{C}$ \\
\hline .1 & 2 & 0.51 & 0.55 & 0.71 & 0.60 & --.-- \\
\hline .2 & 2 & 0.52 & 0.60 & 0.72 & 0.60 &.---- \\
\hline .3 & 2 & 0.55 & 0.65 & 0.74 & 0.60 &.---- \\
\hline .4 & 2 & 0.58 & 0.70 & 0.76 & 0.60 &.---- \\
\hline .5 & 2 & 0.63 & 0.75 & 0.79 & 0.60 & 0.79 \\
\hline .6 & 2 & 0.68 & 0.80 & 0.82 & 0.60 & 0.82 \\
\hline .7 & 2 & 0.75 & 0.85 & 0.86 & 0.60 & 0.86 \\
\hline .1 & 5 & 0.21 & 0.28 & 0.46 & 0.34 &.---- \\
\hline .2 & 5 & 0.23 & 0.36 & 0.48 & 0.34 &.---- \\
\hline .3 & 5 & 0.27 & 0.44 & 0.52 & 0.34 & --.-- \\
\hline .4 & 5 & 0.33 & 0.52 & 0.57 & 0.34 & --.-- \\
\hline .5 & 5 & 0.40 & 0.60 & 0.63 & 0.34 & 0.63 \\
\hline .6 & 5 & 0.49 & 0.68 & 0.70 & 0.34 & 0.70 \\
\hline .7 & 5 & 0.59 & 0.76 & 0.77 & 0.34 & 0.77 \\
\hline .1 & 10 & 0.11 & 0.19 & 0.33 & 0.19 & --.-- \\
\hline .2 & 10 & 0.14 & 0.28 & 0.37 & 0.19 &.---- \\
\hline .3 & 10 & 0.18 & 0.37 & 0.43 & 0.19 &.---- \\
\hline .4 & 10 & 0.24 & 0.46 & 0.49 & 0.19 & 0.49 \\
\hline .5 & 10 & 0.33 & 0.55 & 0.57 & 0.19 & 0.57 \\
\hline .6 & 10 & 0.42 & 0.64 & 0.65 & 0.19 & 0.65 \\
\hline .7 & 10 & 0.54 & 0.73 & 0.74 & 0.19 & 0.74 \\
\hline .1 & 25 & 0.05 & 0.14 & 0.22 & 0.08 & --.-- \\
\hline .2 & 25 & 0.08 & 0.23 & 0.28 & 0.08 & 0.28 \\
\hline .3 & 25 & 0.13 & 0.33 & 0.36 & 0.08 & 0.36 \\
\hline .4 & 25 & 0.19 & 0.42 & 0.44 & 0.08 & 0.44 \\
\hline .5 & 25 & 0.28 & 0.52 & 0.53 & 0.08 & 0.53 \\
\hline .6 & 25 & 0.39 & 0.62 & 0.62 & 0.08 & 0.62 \\
\hline .7 & 25 & 0.51 & 0.71 & 0.71 & 0.08 & 0.71 \\
\hline .1 & 100 & 0.02 & 0.11 & 0.14 & 0.01 & 0.14 \\
\hline .2 & 100 & 0.05 & 0.21 & 0.22 & 0.01 & 0.22 \\
\hline .3 & 100 & 0.10 & 0.31 & 0.31 & 0.01 & 0.31 \\
\hline .4 & 100 & 0.17 & 0.41 & 0.41 & 0.01 & 0.41 \\
\hline .5 & 100 & 0.26 & 0.51 & 0.51 & 0.01 & 0.51 \\
\hline .6 & 100 & 0.37 & 0.60 & 0.61 & 0.01 & 0.61 \\
\hline .7 & 100 & 0.50 & 0.70 & 0.70 & 0.01 & 0.70 \\
\hline
\end{tabular}

Note: The lack of some entries under $H H I^{C}$ comes from the fact that $H H I^{C}$ is not defined when $H H I<H H I_{0}$. 
Furthermore, since $h_{1}>h_{2}>h_{3}$, the difference comparison in (15c) implies the relative difference comparison

$\frac{\sqrt{h_{1}}-\sqrt{h_{2}}}{\sqrt{h_{2}}}<\frac{\sqrt{h_{2}}-\sqrt{h_{3}}}{\sqrt{h_{3}}}$

while that from (15b) could result in an inequality in either direction so that

$\frac{h_{1}-h_{2}}{h_{2}}><\frac{h_{2}-h_{3}}{h_{3}}$.

\section{Value-validity Correction of HHI}

While the HHI lacks Property (P5) necessary for making valid difference comparisons, it appears from (10) and (12) that its square root may have this property at least approximately when the number of firms $n$ is large. In fact, Hirschman (1945) proposed the index $\sqrt{\sum_{i=1}^{n} s_{1}^{2}}$ rather than the HHI in (1) as a measure of concentration. See also Hirschman (1964). However, while Hirschman defined his index for all market-share distributions $S_{n}=\left(s_{1}, \ldots, S_{n}\right)$, a corrected index $H H I^{c}$ will be derived as the square root $\sqrt{H H I}$, but subject to a restriction on $S_{n}$.

As a basis for this derivation, consider the data in Table 1 computed for sets of different values of $\lambda$ and $n$ for the lambda distribution $S_{n}^{\lambda}$ in (3). For the varying $\lambda$ and $n$, the results are given in terms of the values of HHI in (1), $\sqrt{H H I}$, and $C$ in (12) for $S_{n}^{\lambda}$ As pointed out above in (13)-(14), the results in Table 1 also indicate clearly how $H H I$ understates the concentration characteristic, the extent to which depends greatly on $\lambda$ and $n$. These results also show that values of $\sqrt{H H I}$ generally tend to be substantially closer to the values of $C$ in (12) than are those of HHI, unless $n$ and $\lambda$ are very small.

While the expression for $C$ in (12) is based on the particular distribution $S_{n}^{\lambda}$ in (3), the corresponding expression for $C$ based on any market-share distributions $S_{n}=\left(s_{1}, \ldots, s_{n}\right)$ and on the condition in (8) (with equality) is given by

$C\left(S_{n}\right)=\left(1-\frac{1}{n}\right) d^{*}\left(S_{n}\right)+\frac{1}{n}=\left(1-\frac{1}{n}\right) \sqrt{\frac{n H H I\left(S_{n}\right)-1}{n-1}}+\frac{1}{n}$.

Although this measure meets the conditions in (5)(8) for value validity and, being a simple function of $H H I$ and $n$, shares Properties (P1), (P2), and (P4) with $H H I$, it does not share Property (P3). The fact that $C$ lacks the zero-indifference property and is an explicit function of $n$ causes potentially unsatisfactory behavior by $C$ as can be simply exemplified by the two distributions $S_{3}=(0.50,0.30,0.20)$ and $S_{4}=(0.50,0.30,0.10,0.10)$. The result $C\left(S_{3}\right)=0.51$ and $C\left(S_{4}\right)=0.54$ cannot be reasonable since most would agree that $S_{3}$ reveals a higher degree of concentration than does $S_{4}$.

Nevertheless, even though the $C$ in (16) cannot by itself be considered as an acceptable measure of concentration because of its dependence on $n$, it can still serve as a useful reference or basis of comparison because of its other desirable properties, including the value-validity property. Thus, for example, for the lambda distribution $S_{n}^{\lambda}$ in (3), values of $C$ as expressed by (12) can be compared with those of $\sqrt{H H I}$ from (10) for different $\lambda$ and $n$ as given in Table 1.

It is apparent from Table 1 that, while the values of $\mathrm{HHI}$ are less than those of $C$, the values of $\sqrt{H H I}$ exceed those of $C$. In fact, it can be verified for any market-share distribution $S_{n}=\left(s_{1}, \ldots, s_{n}\right)$ that

$H H I\left(S_{n}\right) \leq \mathrm{C}\left(\mathrm{S}_{n}\right) \leq \sqrt{H H I\left(S_{n}\right)}$

with equalities if, and only if, $S_{n}$ equals the $S_{n}^{1}$ in (2) when each of the measures in (17) has the value of 1 . It is seen from Table 1 that the values of $\sqrt{H H I}$ for the distribution $S_{n}^{\lambda}$ tend to approach those of $C$ with increasing $\lambda$ and $n$. Alternatively, for any given $n$, the values of $\sqrt{H H I}$ are seen to approach those of $C$ with increasing $\mathrm{HHI}$-values. It also follows from (16) that if the values of $H H I$ in Table 1 were based on market-share distributions $S_{n}$ (rather than $S_{n}^{\lambda}$ ), the corresponding values of $C$ (and $\sqrt{H H I}$ ) would be those given in Table 1.

It may then be possible to obtain a value-validity correction of $H H I$ by using $\sqrt{H H I}$ under the condition that values of $\sqrt{H H I}$ can only exceed those of $C$ by a small amount less than or equal to some specified limit $\Delta_{0}$. Such a limiting value $\sqrt{\mathrm{HHI}_{0}}$ of $\sqrt{\mathrm{HHI}}$ can be determined by setting $\sqrt{H H I_{0}}-C=\Delta_{0}$ and solving this second-order equation, resulting in

$H H I_{0}=\left[\left(n \Delta_{0}+1\right)-\sqrt{\Delta_{0}(n-1)\left(\Delta_{0} n+2\right)}\right]^{2}$

That is, for any given market-share distribution $S_{n}, \sqrt{H H I}-C \leq \Delta_{0}$ requires $H H I \geq H H I_{0}$. Consequently, a corrected concentration index that approximately meets the value-validity conditions in (5)-(8) can simply be defined as

$H H I^{c}=\left\{\begin{array}{l}\sqrt{H H I} \text { if } H H I \geq H H I_{0} \\ \text { undefined if } H H I<H H I_{0}\end{array}\right.$

for the $H H I_{0}$ given in (18) and for any specified $\Delta_{0}$. 
Since the index defined in (19) satisfies at least approximately the requirement for value validity, this $H H I^{c}$ can be used for making appropriate comparisons between different concentration values. If, however, $H H I<H H_{0}$, only order ("larger than") comparisons are permissible. The degree of approximation depends on, besides $n$, the chosen value of $\Delta_{0}$. By taking partial derivatives of $\mathrm{HHI}_{0}$ in (18), it is found that $\mathrm{HHI}_{0}$ is a strictly decreasing function of $n$ for any given $\Delta_{0}$ and, for any given $n, H H I_{0}$ is strictly decreasing in $\Delta_{0}$ (for $\Delta_{0}<\sqrt{1 / n}(1-\sqrt{1 / n})$, an unacceptably high value).

A suggested choice of $\Delta_{0}$ is $\Delta_{0}=0.05$ for which (18) becomes

$$
H H I_{0}=[(n+20-\sqrt{(n-1)(n+40)}) / 20]^{2}
$$

Thus, the values for which the corrected index in (19) become valid can only differ from $C$ in (16) by at most 0.05 . Generally, this difference can be expected to be substantially lower as indicated by the results in Table 1 for the particular market-share distribution in (3). For some of the cases in Table 1, the $H H I^{c}$ from (19) cannot be used since the values of $H H I$ are less than those of $H H_{0}$ in (20).

\section{Some Real Examples}

As some examples involving real data, consider the market-share data reported by Managerial Econom- ics (2019) based on the U.S. Economic Census. The data in Table 2 are given for a selection of markets (industries) and in terms of the number of firms $n$, the $H H I$ index (for the 50 largest firms), $C$ from (16), and the value-validity corrected index $H H I^{c}$ from (19)-(20).

The $H H I^{c}$ is defined from (19)-(20) for all of the data sets in Table 2 except for Data Set 5 (Primary aluminum production). This result from Data Set 5 is due to the rather low value of $\mathrm{HHI}$ and the fact that $H H I_{0}$ is relatively high because of the small number of firms $(n)$. While $H H I$ and $H H I^{c}$ provide the same results for order ("larger than") comparisons, it can be seen from Table 2 that they provide opposite results for some of the difference comparisons.

In order to exemplify such contradictory results between $H H I$ and $H H I^{c}$ and to simplify the notation, let $h_{i}$ and $h_{i}^{c}$ denote the values of $H H I$ and $H H I^{c}$ in Table 2 for Data Set $i(i=1, \ldots, 8)$. Then, an example of the contradictory results discussed in (15a)-(15e) can be found in Data Sets $h_{2}, h_{4}$, and $h_{8}$ where

$$
\frac{h_{2}+h_{8}}{2}=0.065>h_{4}=0.06>\left(\frac{\sqrt{h_{2}}+\sqrt{h_{8}}}{2}\right)^{2}=0.05
$$

and hence $h_{2}-h_{4}=0.06>h_{4}-h_{8}=0.05$, whereas, in terms of $h^{c}=\sqrt{h}, h_{2}^{c}-h_{4}^{c}=0.10<h_{4}^{c}-h_{8}^{c}=0.14$.

Table 2

\begin{tabular}{|c|c|c|c|c|c|c|c|}
\hline $\begin{array}{l}\text { Data } \\
\text { set }\end{array}$ & Market (Industry) & Nacis code & $n$ & $H H I$ & $C$ & $H H I_{0}$ & $H H I^{C}$ \\
\hline 1 & Breakfast cereal & 31123 & 48 & 0.24 & 0.48 & 0.04 & 0.49 \\
\hline 2 & Coffee and tea & 31192 & 215 & 0.12 & 0.34 & 0.005 & 0.35 \\
\hline 3 & Luggage & 316991 & 270 & 0.14 & 0.37 & 0.004 & 0.37 \\
\hline 4 & Soap and cleaning products & 32561 & 1,583 & 0.06 & 0.24 & 0.001 & 0.24 \\
\hline 5 & Primary aluminum production & 331312 & 13 & 0.12 & 0.28 & 0.15 &.--- \\
\hline 6 & Constructive machinery & 33312 & 723 & 0.10 & 0.32 & 0.000 & 0.32 \\
\hline 7 & Automobiles & 33611 & 253 & 0.29 & 0.54 & 0.004 & 0.54 \\
\hline 8 & Medical equipment & 3911 & 12,123 & 0.01 & 0.10 & 0.001 & 0.10 \\
\hline
\end{tabular}

Values of the Quantities in (1), (16), (19), and (20) for a Sample of Manufacturing Industries from the U.S. Economic Census

Source: Managerial Economics (2019). 
When considering relative differences, $100\left(h_{2}-h_{4}\right) / h_{4}=100 \%$ as compared to $100\left(h_{4}-h_{8}\right) / h_{8}=500 \%$ and $100\left(h_{2}^{c}-h_{4}^{c}\right) / h_{4}^{c}=41 \%$ as compared to $100\left(h_{4}^{c}-h_{\mathrm{R}}^{c}\right) / h_{\mathrm{8}}^{c}=145 \%$.

Other examples of contradictory results involving $H H I$ and $H H I^{c}$ can be seen from Data Sets 1 , 3 , and 4 where $h_{1}-h_{3}=0.10>h_{3}-h_{4}=0.08$, whereas $h_{1}^{c}-h_{3}^{c}=0.12<h_{3}^{c}-h_{4}^{c}=0.13$.

Similarly, when comparing the results from $\mathrm{HHI}$ and $H H I^{c}$ for Data Sets $1,4,6$, and 7 , it is seen that $h_{6}-h_{4}=0.04<h_{7}-h_{1}=0.05$, but $h_{6}^{c}-h_{4}^{c}=0.08>h_{7}^{c}-h_{1}^{c}=0.05$. Also, $h_{1}-h_{2}>h_{2}-h_{8}$, whereas $h_{1}^{c}-h_{2}^{c}<h_{2}^{c}-h_{8}^{c}$.

It is also seen from the results in Table 2, when $H H I^{c}$ is defined as in (19)-(20), its values are generally much closer to those of $C$ in (16) than the maximum difference $\Delta_{0}=0.05$. If $H H I^{c}$ is used to predict $C$, then the coefficient of determination, when properly computed (Kvålseth, 1985) is found for the 7 data points in Table 2 to be $R^{2}=\sum\left(C-H H I^{c}\right)^{2} / \sum(C-\bar{C})^{2}=0.998$. That is, nearly all of the variation (99.8\%) of $C$ is explained by the fitted model $\hat{C}=H H I^{c}$. For the 23 data points in Table 1 for which $H H I^{c}$ is defined, the equivalent value of $R^{2}$ is found to be $R^{2}=0.989$.

\section{Concluding Comments}

The $C$ in (16) with its value-validity and other desirable properties would have been an excellent substitute for $H H I$ had it not been for the fact that $C$ lacks the zero-indifference property (Property (P3)). In fact, $C$ is an explicit function of the number of firms $n$ within a market (industry). The proposed $H H I^{c}$ in (19)-(20) takes on values that closely approximate those of $C$, but $H H I^{c}$ does have the zero-indifference property (as does $H H I$ ). The extent to which $H H I^{c}$ is affected by $n$ is limited to whether or not this index is defined for any given market-share distribution $S_{n}=\left(s_{1}, \ldots, S_{n}\right)$, but $n$ does not affect the value of $H H I^{c}$ for any given $S_{n}$.

While $H H I$ and $H H I^{c}$ share Properties (P1)-(P4), their important difference is that $H H I^{c}$, but not $H H I$ meets the conditions for value validity to a high degree of approximation because of the close correspondence between its values and those of the index $C$ in (16), which has Property (P5). Consequently, while the proper use of HHI should be limited to order ("greater than" or "smaller than") comparisons between HHI values, values of $H H I^{c}$ can also reasonably be compared in terms of actual, absolute, or relative differences. Thus, whenever values of the $H H I$ and $n$ are such that those of $H H I^{c}$ can be defined as in (19), HHI values and comparisons can reasonably be expected to provide true and realistic representations about market concentration. This correction of $\mathrm{HHI}$ eliminates potentially invalid and misleading results and interpretations of market concentrations.

The concentration measures discussed above are all based on the market-share distribution $S_{n}=\left(s_{1}, \ldots, S_{n}\right)$. However, in many real situations the market shares for all firms within a market or industry are not known or reported. Quite typically, the market shares of the smaller firms are simply grouped into an "all others" category. Such data limitation can cause a restriction when measuring concentration (e.g., Busu, 2020; Kvålseth, 2018). Depending, of course, on the extent to which such reported data are limited, excluding the very small market shares from the computation of $\mathrm{HHI}$ in (1) and $H H I^{c}$ in (19) has rather limited effect since small market shares $\left(s_{i}\right)$ are assigned low weights. However, in the case of the index $C$ in (16), such data limitation effect may be substantial because of the effect on the size of the market $n$.

One way to account for the effect of such incomplete market-share data is based on the bounds for the lacking data as follows. Thus, consider the following expression for $\mathrm{HHI}$ :

$H H I=\sum_{i=1}^{m} s_{i}^{2}+\sum_{i=m+1}^{n} s_{i}^{2}$

where $S_{m+1}, \ldots, S_{n}$ are the market shares of the $n-m$ smallest firms that may not be available. Upper and lower bounds on $\sum_{i=m+1}^{n} s_{i}^{2}$ can be expressed as follows (Kvålseth, 2018; Busu, 2020):

$A=(n-m)^{-1}\left(\sum_{i=m+1}^{n} s_{i}\right)^{2} \leq \sum_{i=m+1}^{n} s_{i}^{2} \leq s_{m} \sum_{i=m+1}^{n} s_{i}=B$

where $\sum_{i=m+1}^{n} s_{i}$ is the combined market share of the ( $n-m)$ smallest firms and $s_{m}$ is the market share of the $m$-th largest firm. See, also, Naldi and Flamini (2017). Thus, when values of $H H I$ are based on the first term in (21), the bounds in (22) provide a measure of the potential tolerance or error limits of $H H I$ and hence of $H H I^{c}$ in (19)

While $B$ in (22) does not depend on the total number of firms $n$, the lower bound $A$ does depend on $n$, which is typically not reported when the smallest market shares are grouped together. However, based on $B$ in (22), it is found that the measure

$H H I^{\prime}=\sum_{i=1}^{m} s_{i}^{2}+s_{m} \sum_{i=m=1}^{n} s_{i} / 2$

provides a good approximation to $H H I$ for both simulated and real market-share data (Kvålseth, 2018). This HHI'would then also replace HHI in the expres- 
sion for $H H I^{c}$ in (19). However, for some reported data, the second term in (23) may be so small that it can effectively be ignored for practical purposes.

An advantage that HHI has over other concentration indices is that it emerges from a well-known theoretical model of market performance and competition. Specifically, under Cournot competition and using the market shares as the weights, the weighted mean of the firms' price-cost margins (PCM) becomes equal to $H H I$ divided by the absolute value of the market demand elasticity (e.g., Martin, 2002, pp. 337-338; Carlton and Perloff, 1994, p. 375). However, the underlying theoretical model is not uniformly accepted. Boone (2008), for instance, has argued that the theoretical basis for PCM as a measure of competition is not a robust one. Instead, he proposes the relative profit differences (RPD) as a more robust theoretical foundation for a measure of industry competition. However, Boone (2008) does not consider the effect of the RPD competition model versus the PCM model on $H H I$ itself or if the RPD leads to some alternative index as a function of the market shares $s_{1}, \ldots, s_{n}$.

Irrespective of the theoretical basis of $H H I$, this index has become the most popular measure of market (industry) concentration. However, as discussed in this paper, caution needs to be exercised when using $H H I$ in order to avoid potentially incorrect and misleading results. A simple corrected form of this index, the $H H I^{c}$ in (19), permits different types of concentration comparisons to be made as true, valid, or realistic representation of real market situations. The corrected form $H H I^{c}$ does, however, have the restriction given in (19). Future research ought to explore some alternative index formulation that would have all of the desirable properties of $H H I^{c}$ and be applicable to all market-share distributions without any restriciton.

\section{References}

Ambarci, N.,\& Katzman, B. (2015). A new industry concentration index. Economic Papers, 34(4), 222228. https://doi.org/10.1111/1759-3441.12122

Bikker, J. A., \& Haaf, K. (2002). Measures of competition and concentration in the banking industry: a review of the literature. Economic \& Financial Modelling, 9(2), 53-98.

Boone, J. (2008). A new way to measure competition. The Economic Journal, 118(531), 1245-1261. https:// doi.org/10.1111/j.1468-0297.2008.02168.x Busu, M. (2020). A market concentration analysis of the biomass sector in romania. Resources, 9(6), 64.
https://doi.org/10.3390/resources9060064Calkins, S. (1983). The new merger guidelines and the Herfindahl-Hirschman index. California Law Review, 71(2), 402-429.

Carlton, D. W., \& Perloff, J. M. (1994). Modern Industrial Organization ( $2^{\text {nd }}$ ed.). Harper Collins College Publishers.

Chakravarty, S. R., \& Eichhorn, W. (1991). An axiomatic characterization of a generalized index of concentration. The Journal of Productivity Analysis, 2, 103112. https://doi.org/10.1007/BF00156341

Encaoua, D., \& Jacquemin, A. (1980). Degree of monopoly, indices of concentration and threat to entry. International Economic Review, 21(1), 87-105. https:// doi.org/10.2307/2526242

European Union. (2004). Guidelines on the assessment of horizontal mergers. Official Journal C-031, 05/02/2004, 0005-0018.

Gaughan, P. A. (2011). Mergers, Acquisitions, and Corporate Restructuring, $5^{\text {th }}$ ed. Wiley.

Ginevičius, R., \& Čirba, S. (2007). Determining market concentration. Journal of Business Economics and Management, 8(1), 3-10. https://doi.org/10.1080/1 6111699.2007.9636147

Naldi, M., \& Flamini, M. (2017). Censoring and distortion in the Hirschman-Herfindahl index computation. Economic Papers, 36(4), 401-415. https://doi. org/10.1111/1759-3441.12187

Hall, M., \& Tideman, N. (1967). Measures of concentration. Journal of the American Statistical Association, 62(317), 162-168.

Hannah, L., \& Kay, J. A. (1977). Concentration in modern industry. Macmillan.

Herfindahl, O. C. (1950). Concentration in the steel industry [Unpublished Ph.D. dissertation]. Columbia University.

Hirschman, A. O. (1945). National power and the structure of foreign trade. University of California Press.

Hirschman, A. O. (1964). The paternity of an index. The American Economic Review, 54, 761-762.

Kvålseth, T. O. (1985). Cautionary note about $\mathrm{R}^{2}$. The American Statistician, 39, 279-285. https://doi.org/ 10.1080/00031305.1985.10479448

Kvålseth, T. O. (2011). The lambda distribution and its applications to categorical summary measures. Advances and Applications in Statistics, 24, 83-106. https://doi.org/10.1073/pnas.0711411105

Kvålseth, T. O. (2015). Evenness indices once again: critical analysis of properties. SpringerPlus, 4(1), 1-12. . https://doi.org/10.1186/s40064-015-0944-4, 1-12.

Kvålseth, T. O. (2018). Relationship between concentration ratio and Herfindahl-Hirschman index: A 
re-examination based on majorization theory. Heliyon, 4(10), e00846. https://doi.org/10.1016/j.heliyon.2018.e00846

Managerial Economics (2019). Tutorial: Census measures of market concentration. (Accessed October 8,2019 ).

Marshall, A. W., Olkin, I., \& Arnold, B. C. (2011). Inequalities: Theory of Majorization and its Applications, $2^{\text {nd }} \mathrm{ed}$. Springer.

Martin, S. (2002). Advanced Industrial Economics, $2^{\text {nd }} e d$. Blackwell.

Ukav, I. (2017). Market structures and concentration measuring techniques. Asian Journal of Agricultural Extension, Economics \& Sociology, 19(4), 1-16. https://doi.org/10.9734/AJAEES/2017/36066.

U.S. Department of Justice and the Federal Trade Commission. (2010). Horizontal Merger Guidelines (August 19, 2010). 